\title{
Inflammatory Psuedotumor of Lung as a Space-Occupying Lesion in the Chest: A Diagnostic Challenge
}

\author{
Sriram Vijayapuri ${ }^{1}$, Sushma Krishna ${ }^{2}$, Shashidhar Buggi ${ }^{1}$ \\ Departments of ${ }^{1}$ Cardio-Vascular-Thoracic Surgery and ${ }^{2}$ Microbiology, SDS Tuberculosis Research Centre \& Rajiv Gandhi Institute \\ of Chest Diseases, Bangalore, India.
}

\section{Corresponding Author:}

Dr. Sushma Krishna

Email: drsushmakrishna@gmail.com

This is an Open Access article distributed under the terms of the Creative Commons Attribution License (creativecommons.org/ licenses/by/3.0).

Received Accepted

Published

May 14, 2019

August 8, 2019

August 20, 2019

\begin{abstract}
Background: Inflammatory pseudotumor of the lung is a rare benign tumor of the lung seen in pediatric age group. Case Report: We describe two cases of adolescents who presented with repeated episodes of recurrent respiratory tract infection from over six years. Chest X-ray and computerized tomography findings were provisionally indicative of intraparenchymal bronchopulmonary sequestration in the left lower lobe and right middle lobe respectively. Left thoracotomy with enucleation was carried out in one, while the other underwent right thoracotomy with debulking of the tumor. Excision biopsy in both confirmed the unanticipated diagnosis of inflammatory pseudotumour of the lung. Conclusion: This is a rare inflammatory non-neoplastic condition commonly affecting children and young adults, often posing a diagnostic challenge clinically, radiologically and pathologically.
\end{abstract}

Keywords: Biopsy, Broncopulmonary Sequestration, Chest X-ray, Granuloma, Lung, Plasma Cells.

\section{Introduction}

Inflammatory pseudotumor of the lung is a rare benign tumor of the lung which usually presents in the pediatric or adolescent age group. It has been addressed by numerous names such as plasma cell granuloma, fibrous histiocytoma, or fibroxanthoma because of its predominant cellular component. Most commonly follows a benign course but can rarely be recurrent, invasive, or undergo malignant transformation. Only few cases have been reported from India in the last decade [1-6] and we communicate our experience with the first two cases encountered.

\section{Case Reports}

\section{Case 1}

A fourteen-year-old girl, without any known comorbidity presented with complaints related to lower respiratory tract infections from the last six years, about 4-5 times a year for which she had been treated with antibiotics, analgesics and expectorant. With the previous episode, she was investigated with chest X-ray and CT thorax. Chest X-ray revealed well circumscribed left lower zone paracardiac opacity while CT-thorax showed a well-defined hypodense circumscribed lesion $(7 \times 6.5 \times 6 \mathrm{~cm})$ in the left lower lobe, surrounded by lung parenchyma with areas of air density in the center. Lesion showed enhancement on contrast with systemic arterial connection (from aorta) and was reported as an intraparenchymal bronchopulmonary sequestration. Pleural spaces were clear.

She was referred to our state reference center for further management. Other associated anomalies such as trachea-esophageal fistula, foregut anomalies or cardiac malformation were 
not seen. Sputum microscopy was negative for acid fast bacillus (AFB). The hematological and biochemical results were within normal range. On physical examination, she was poorly built and nourished with decreased air entry on the left basal area. Patient underwent left thoracotomy with enucleation (postero-lateral, $6^{\text {th }}$ space). Examination of the lesions that was visible on the diaphragmatic surface of the lower lobe revealed a firm, almost lung like mass, well encapsulated and partially adherent to surrounding lung parenchyma. The mass was excised in-toto [Fig.1] and biopsy was sent for histopathological examination. Postoperative period was uneventful and patient was discharged from hospital on the $8^{\text {th }}$ day. A check Xray after two weeks showed good lung expansion with no further clinical symptoms. Histopathology report showed sheets of plasma cells admixed with few histiocytes and lymphocytes suggestive of inflammatory pseudotumor of lung and was confirmed by immunohistocytochemistry [Fig.2].

\section{Case 2}

A seventeen year old male also presented with repeated episodes of upper respiratory tract infections from over six years with an episode of hemoptysis $(250 \mathrm{ml})$ for which he was referred to our hospital. Chest X-ray revealed circumferential lesion in the right mid zone while CT-thorax showed an eccentric, well defined heterogeneously enhancing areas in the middle mediastinum causing compression of right main bronchus with loss of fat planes between the mass and bronchial wall (dimensions of $3 \mathrm{~cm} \times 1 \mathrm{~cm} \times$ $1 \mathrm{~cm}$ ) without parenchymal lesion or mediastinal lymphadenopathy. Patient underwent right thoracotomy with debulking of the tumor. It was a highly vascular tumor in the fissure between upper and middle lobe of right lung. Removal of the whole tumor was achieved, hemostasis was secured and biopsy was sent for histopathological examination. Post-operative period was uneventful and patient was discharged from hospital on the $7^{\text {th }}$ day. Biopsy report showed proliferation of spindle cells

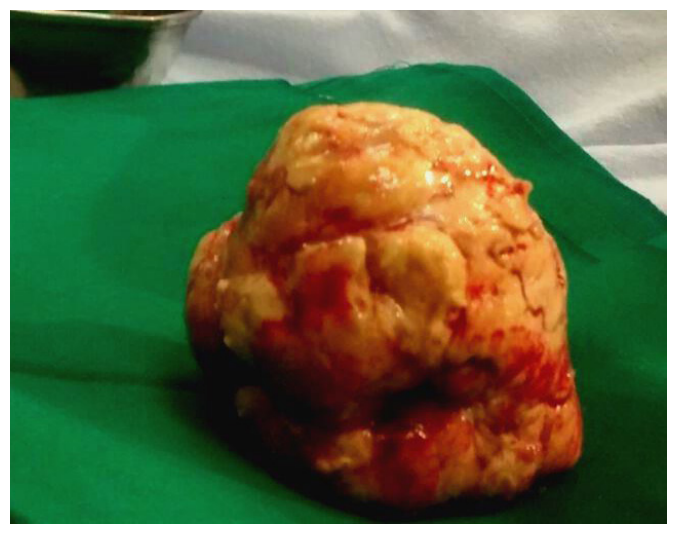

Fig.1: Gross picture of the excised mass.

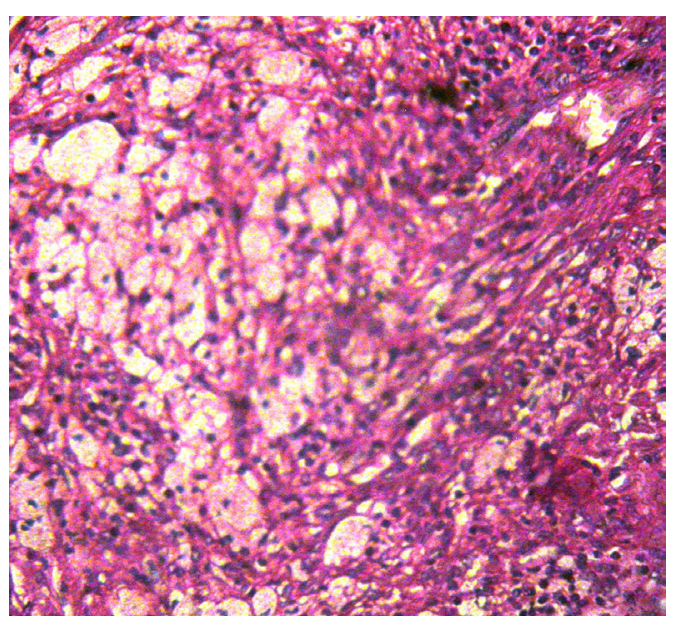

Fig.2: Microscopy showing plasma cells admixed with few histiocytes and lymphocytes.

in a myxoid background with cells having large, vesicular nucleoli, pink cytoplasm and elongated processes. Good number of inflammatory cells (predominantly plasma cells) were seen with areas of hemorrhage and many blood vessels.

\section{Discussion}

Inflammatory pseudotumor is one of the surgical conditions which pose a challenge to diagnose and mimics a malignant process. It accounts for $<1 \%$ of all tumors of the lung and airways. Little is known about its natural history, various postulated theories and pathophysiological mechanisms attribute it to a metabolic disturbance, trauma or surgery, organized cellular growth developing in association with 
pulmonary infection, auto-immune mechanism, viral origin or antigen-antibody interaction to no longer identifiable etiological agent [7]. Specific infectious agents such as Mycobacteria in association with spindle cell tumor and Nocardia and Mycoplasma in pulmonary pseudotumors have been incriminated [8].

Being rare to any organ such as brain or liver, it develops most commonly in the lung [9] and has the propensity to involve orbit, stomach, testis, esophagus, liver, spleen, pancreas, kidney, adrenal gland, retro-peritoneum, diaphragm, mesentery, bladder, heart, thyroid, tonsil, fourth ventricle, spinal cord meninges, central nervous system, maxillary sinus, naso-pharynx, larynx and trachea [8]. Low index of suspicion with the biopsies showing only inflammatory cells adds on to the diagnostic dilemma for both the clinician and pathologist respectively [10]. The differential diagnosis microscopically includes lymphoma, sarcoma, and fibrosis.

The pseudotumor has an increased predilection to develop in children. The clinical symptoms too stand non-specific. Generally, patient presents with cough, chest pain, dyspnea and hemoptysis, may even be asymptomatic. Fever and clubbing have been noted particularly in younger patients. Weight loss and fatigue have been also described [7]. A history of prior infection could be obtained in about one third of patients as in both of these patients. Laboratory investigations may show elevated ESR, anaemia, thrombocytosis, and hypergammaglobulinemia, which characteristically resolve after the lesion is excised [8]. However, the laboratory data are usually not of much help in diagnosis of inflammatory pseudotumor as was in these cases.

The radiographic and $\mathrm{CT}$ appearance of inflammatory pseudotumor is quite variable and non-specific. CT scan most commonly demonstrates a mass of heterogeneous attenuation as in both of these cases. Variable enhancement patterns have been described on contrast enhanced CT scans, including non-enhancement, mild enhancement, heterogeneous enhancement and peripheral enhancement. Variable calcification patterns have been described on CT scans including punctate, dense, flocculent and curvilinear [11]. Because inflammatory pseudotumors mimic malignant tumors radiologically, the radiologist should be familiar with this entity and help avoid unnecessary radical surgery when possible.

Resection of the lesion is the preferred treatment. The unusual gross appearance in situ (i.e. well encapsulated and tethered) in the first case specifically allowed an enucleation rather than regular formal anatomical lung resection. However, in cases of incomplete resection or recurrence, nonsurgical treatment likes radiotherapy, chemotherapy and steroids have been successfully used. The role of antibiotics is uncertain, neither bacteria nor fungi have been grown in tissue cultures in any of the resected specimens. As preoperative investigation is not diagnostic, excision of the mass is imperative in order to exclude malignancy. Complete resection, when possible, is safe and leads to excellent survival and remains the key to prevent recurrence [12]. The prognosis of these rare tumors is excellent after complete surgical excision. There is a low incidence of recurrence with long-term follow-up after complete removal of the mass.

\section{Conclusion}

We conclude that inflammatory pseudotumor of the lung is rare. They often occur in children and adolescents, can grow to a large size, and may be often locally invasive with symptoms requiring significant pulmonary resection. Complete resection, when possible, is safe and leads to excellent survival. The radiographic and CT appearance of inflammatory pseudotumor is quite variable and nonspecific. Therefore, when clinical presentation of pulmonary neoplasm or infection is atypical or problematic, it is important for 
clinicians to consider the diagnosis of inflammatory pseudotumor and to be aware of the variety of its different radiographic and CT manifestations.

Contributors: SV: manuscript editing, patient management; SK: manuscript writing, patient management; SB: critical inputs into the manuscript and patient management. SK will act as a study guarantor. All authors approved the final version of this manuscript.

Funding: None; Competing interests: None stated.

\section{References}

1. Singh RS, Dhaliwal RS, Puri D, Behera D, Das A. Inflammatory pseudotumor of the lung: Report of a case and review of literature. Indian J Chest Dis Allied Sci. 2001;43:231-234.

2. Patel SB, Shah DM, Goswami KG, Shah SR, Shah PM. Inflammatory pseudotumor of lung, a case report and review of literature. Ind J Radiol Imag. 2006;16:117118.

3. Prasad MV, Thankachen R, Parihar B, Shukla V. Inflammatory pseudotumour of the lung. Interactive Cardiovascular and Thoracic Surgery. 2004;3:323-325.

4. Ray A, Suri JC, Bhattacharya D, Gupta A. Bronchoscopic resection of endobronchial inflammatory myofibroblastic tumor: A case report and systematic review of the literature. Lung India. 2014;31:172-175.
5. Bhatnagar S, Nigam S, Mandal AK. Indian J Chest Dis Allied Sci. 2001;43:55-57.

6. Patankar T, Prasad S, Shenoy A, Rathod K. Pulmonary inflammatory pseudotumor in children. Australas Radiol. 2000;44:318-320.

7. Cerfolio RJ, Allen MS, Nascimento AG, Deschamps C, Trastek VF, Miller DL, et al. Inflammatory pseudotumors of the lung. Ann Thorac Surg. 1999;67:933-936.

8. Narla LD, Newman B, Spottswood SS, Narla S, Kolli R. Inflammatory psuedomtumor. Radiographics. 2003; 23:719-729.

9. Tan-Liu NS, Matsubara O, Grillo HC, Mark EJ. Invasive fibrous tumor of the tracheobronchial tree: clinical and pathological study of seven cases. Hum Pathol. 1989; 20:180-184.

10. Copin MC, Gosselin BH, Ribet ME. Plasma cell granuloma of the lung: difficulties and prognosis. Ann Thorac Surg. 1996;61:1477-1482.

11. Agrons GA, Rosado-de-christenson ML, Kirejczy KWM, Conran RM, Stocker JT. Pulmonary inflammatory pseudotumor: radiologic features. Radiology. 1998;206:511-518.

12. Venizelos I, Papathomas T, Anagnostou E, Tsanakas J, Kirvassilis F, Kontzoglou G. Pediatric inflammatory myofibroblastic tumor of the trachea: A case report and review of the literature. Pediatr Pulmonol. 2008;43:831-835. 